\title{
Real-time Mobile-to-Mobile Stethoscope for Distant Healthcare
}

\author{
Bing-Yuh Lu, ${ }^{* * *}$, Ling-Yuan Hsu**, Huey-Dong $\mathrm{Wu}^{* * * *}$, \\ Meng-Lun Hsueh****, San-San Sing*****, Rui-Han Tang*, \\ Mei-Ju Su******, Jhen-Cheng Wang*, Jin-Shin Lai******* \\ *Department of Electronic Engineering, Tungnan University, New Taipei City, Taiwan, ROC \\ ** Department of Information Management, St. Mary's Junior College of Nursing, Medicine, and Management, \\ YiLan, Taiwan, ROC \\ ${ }^{* * *}$ Department of Integrated Diagnostics and Therapeutics, ${ }^{* * * * * * *}$ Department of Rehabilitation \\ National Taiwan University Hospital, Taipei, Taiwan \\ ***** Department of Electronic Engineering, Hwa-Hsia Institute of Technology, New Taipei City, Taiwan, ROC \\ ${ }^{* * * * * * *}$ Department of Business Administration, National Taipei College of Business, Taipei, ROC \\ ${ }^{* * * * * * *}$ Department of Biomedical Engineering, Yuanpei University, HsinChu, Taiwan, ROC \\ benjaminee777@hotmail.com, lyhsuesmc.edu.tw, hdwuntuh@ntu.edu.tw, \\ elic0912@gmail.com, sing@ntcb.edu.tw, schedule730@gmail.com, \\ merri1024@gmail.com, jcwang@mail.tnu.edu.tw, jslai@ntu.edu.tw
}

\begin{abstract}
This study presented to insert a small-scaled microphone of the ear-set of a smart mobile (Amazing A6, Taiwan MobileTM) into an eartip of the stethoscope, the sound can be transmitted by the mobile when it dialed to another phone or mobile. In this study, we employed another smart phone (Galaxy R GT19103, SamsungTM) to be the receiver. The results were showed by spectrogram which demonstrated the components of frequencies of the sounds. Finally, we proposed to improve the study by database.
\end{abstract}

Keywords - acoustic signal, lung sound, heart sound, mobile, smart phone., distant healthcare.

\section{INTRODUCTION}

The real-time distant healthcare is always crucial to the patients, especially, to detect the heart and lung sound. The first stethoscope was invented by a French physician, Rene Laennec, in 1816 [1]. The purpose of auscultation is to listen to sounds inside the body. The auscultatory locations are heart, lungs, gastrointestinal tracts and so on. Therefore, auscultation can detect the physiological sounds from circulatory, respiratory, and digestive system. Doctors follow the standards of procedures of auscultation for diagnosis.

Wheezes are continuous and audible. Wheezes are due to airway wall oscillations or bronchus narrowing generated by.
The word, continuous, means that the wheezes durations are longer than $250 \mathrm{~ms}$ and the frequencies of $200 \mathrm{~Hz}$ [2]. The new definition of the present CORSA guidelines, dominant frequency of wheezes is $100 \mathrm{~Hz}$ greater and the duration 100 ms or more [3]. Rhonchi are defined as low pitch continuous sounds with a dominant frequency of about $200 \mathrm{~Hz}$ or less. Some investigators have proposed that the term rhonchi should be abandoned and modified to use only low pitch wheezes $[4,5]$. The conditions related to wheezes includes bronchospasm, airway thickening caused by mucosal swelling or muscle hypertrophy, inhalation of a foreign object, tumor, secretions, or dynamic airway compression [6]. Wheezes may be monophonic or polyphonic. Severe status asthmatics patients could be heard the wheezes random monophonic. Many persons have a fancy for the modern smart phone, such as Galaxy Note III (Samsung), New One (HTC), iPhone 5 (Apple), and so on, because there are so many new applications(APP) in the new generation of the smart phones. Based on the new wireless communication technologies which included blutooth technology, the 3 or $4 \mathrm{G}$ mobile communication system, and modern electronic stethoscope, we developed a mobile based real-time stethoscope (MBRS) for distant healthcare to improve the convenience of the realtime auscultation for the patients. 

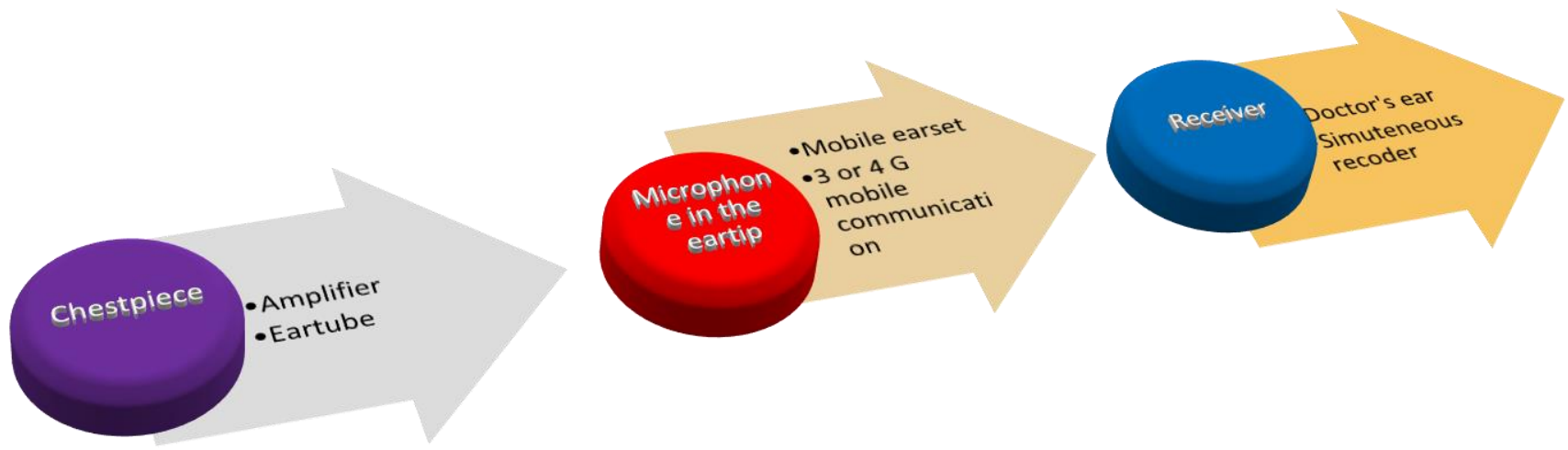

Fig. 1. the block diagram of the MBRS.

\section{METHOD}

Figure 1 presented the block diagram of the MBRS. The input was the electronic stethoscope (3MTM Littmann ${ }^{\circledR}$ Model 3200). The stethoscope was designed by the technologies of ambient noise reduction, frictional noise dampening, electronic amplification, Bluetooth ${ }$ data transfer, and all-new user interface. The detected sound can be transferred by the Bluetooth ${ }^{\circledR}$ wireless technology to the computer which needed to install the computer software "StethAssist" (3MTM Littmann $\left.{ }^{\circledR}\right)$ to show and save the visualized sound signals. We inserted a small-scaled microphone of the ear-set of a smart mobile (Taiwan mobileTM, Model: Amazing A6) into an eartip of the stethoscope, the sound can be transmitted by the mobile when it dialed to another phone or mobile. In this study, we employed another smart phone (Galaxy R GT19103, SamsungTM) to be the receiver. If the receiver was embedded a real-time recorder which might be a circuit or a computer software, the detecting sounds can be saved for the further analysis.

As we know, the calibration always needs a golden standard to be the comparison of the measured signals. However, the real heart, and lung sounds are not easy to be defined, so we take the reliability of the well-known brand of the 3MTM Littmann ${ }^{\circledR}$, and the modern Bluetooth ${ }^{\circledR}$ data transfer technology to define the real sound from chestpiece. The spectrogram is a convenient tool for speech recognition $[7,8]$. The same applications can be employed in the respiration acoustic signals. In general, the spectrogram can be $\boldsymbol{I}(\boldsymbol{k}, \boldsymbol{f})=\sum_{\boldsymbol{k}=\mathbf{0}}^{\left[\frac{\boldsymbol{\ell}}{\boldsymbol{\ell} \boldsymbol{t}_{\boldsymbol{o}}}\right]} \boldsymbol{S F}\left(\boldsymbol{s}^{2}(\boldsymbol{\tau})\left[\boldsymbol{u}\left(\boldsymbol{k} \boldsymbol{\ell}+\boldsymbol{t}_{\boldsymbol{o}}\right)-\boldsymbol{u}((\boldsymbol{k}+\mathbf{1}) \boldsymbol{\ell}+\right.\right.$ $\left.\left.\left.t_{o}\right)\right], \boldsymbol{\ell}, \boldsymbol{t}_{o}, F, \Delta f\right)$ where $\mathrm{k}$ denotes the time in the spectrogram, $\mathrm{f}$ is frequency, and $\mathrm{I}$ is the intensity of the signal spectrum. $\boldsymbol{S \mathcal { F }}\left(\mathrm{s}, \boldsymbol{\ell}, \boldsymbol{t}_{\boldsymbol{o}}\right)$ represents the short-time Fourier Transform (STFT) of the signal s of the length $\boldsymbol{\ell}$ with the overlapped length $\boldsymbol{t}_{\boldsymbol{o}}$, and T for the length of the signal s. F is the maximum frequency of STFT. $\Delta \boldsymbol{f}$ is the frequency resolution of the spectrogram. The diagram of the spectrogram is an alignment of individual components of $\mathrm{I}(\mathrm{k}, \mathrm{f})$, where the $\mathrm{X}$ axis represents $\mathrm{k}$, the $\mathrm{Y}$ axis represents $\mathrm{f}$, and the $\mathrm{Z}$ axis represents $\mathrm{I}$.

\section{III.RESULTS}

Figure 2 showed the experimental devices of this study. A microphone inserted in the one of the eartubes of the electronic stethoscope (3MTM Littmann ${ }^{\circledR}$ Model 3200) to send the sound to the mobile (Amazing A6, Taiwan

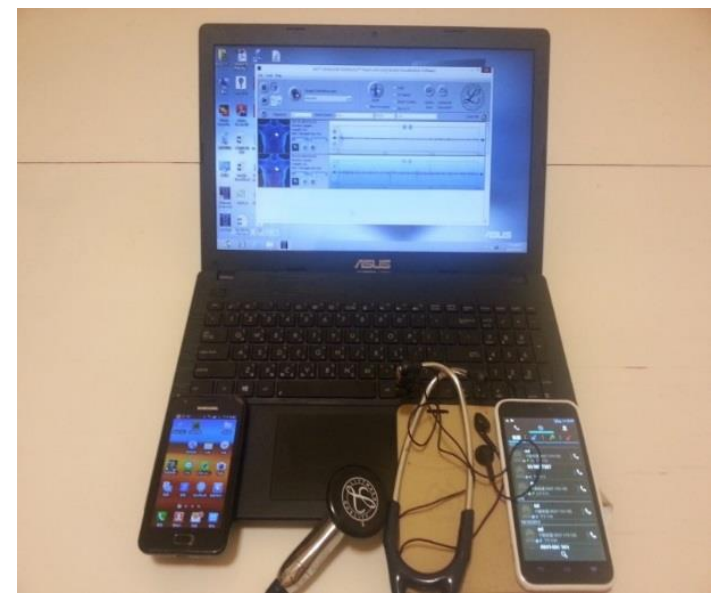

Fig. 2.The transmitter of the system. 
MobileTM). Concurrently, the digitalized data of sound was transferred to the notebook (X551, ASUSTM) which was installed computer software "StethAssist" (Littmann ${ }^{\circledR}$, $3 \mathrm{MTM}$ ) to show and save the visualized sound signals in time domain. The wave shape, and its spectrogram of the lung sound was shown in Fig.3, and Fig.4 which were the cases of normal subject and getting cough subject, respectively. From the differences of the two cases, we can ensure the reliability of the system. The spectrogram in Fig. 4 presented the higher frequency components were more than that in Fig. 3. If fact, the subject in Fig. 4 has gotten a cold. He had a headache, and a running nose. By the signal transmission, the heart and lung sound can be listened by the receiver of smart phone (Galaxy R GT19103, SamsungTM). The quality of the sound can be improved by listening of using the earphone which was the original auxiliary of the smart phone.

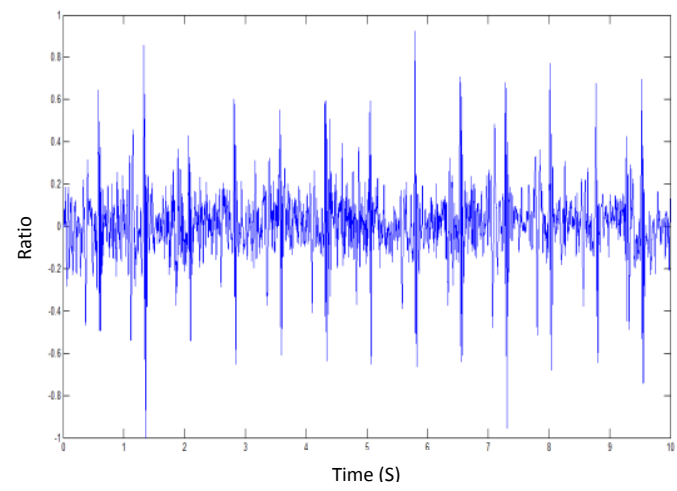

(a)

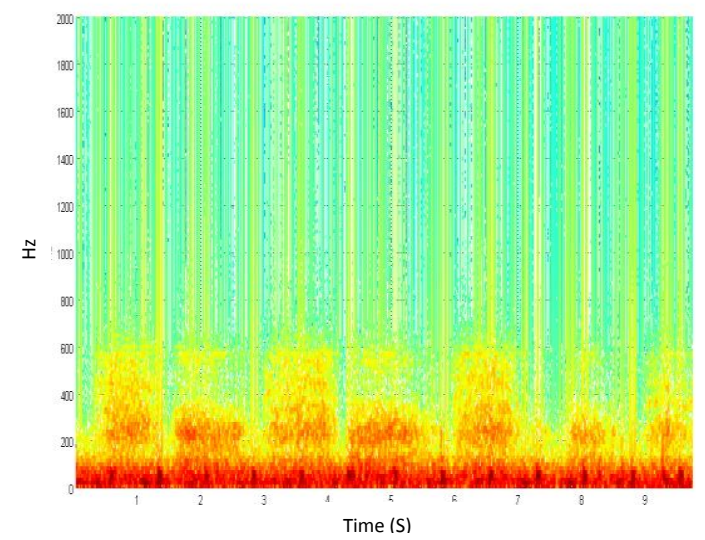

(b)

Fig 3. Normal subject: (a) the recorded lung sound, (b) corresponding spectrogram

\section{IV.DISCUSSION}

An excellent stethoscope can aid the doctors to detect the problems of hearts or air ways of the patients. However, the abnormal sounds are not always occur. When the patients go to hospital, their chief complains were frequently described by the histories of their daily-life. This system of this study supports the possibility of the real-time diagnosis to enhance the precision of the auscultation.

The electronic stethoscope amplified the detecting sound with high fidelity by electronic amplifiers. Although, we heard the sound aloud, the quality of the sound was guaranteed. This is the key device of this study. Because the heart, and lung sounds can aid the diagnosis of cardiac murmur, mitral regulation, wheeze, and other cardiovascular and respiration diseases. The sampling rates of the whole communication processes were listed as follows: The electronic stethoscope was $4 \mathrm{KHz}$ of sampling rate, and the mobile communication was $8 \mathrm{KHz}$ of sampling rate. In our

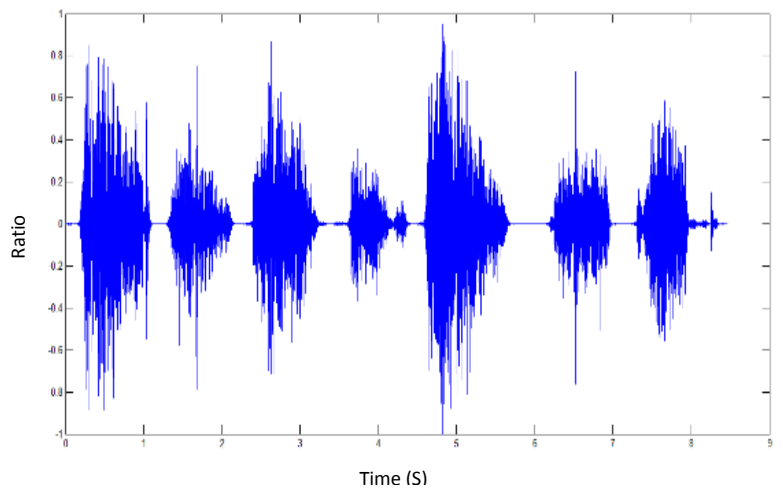

(a)

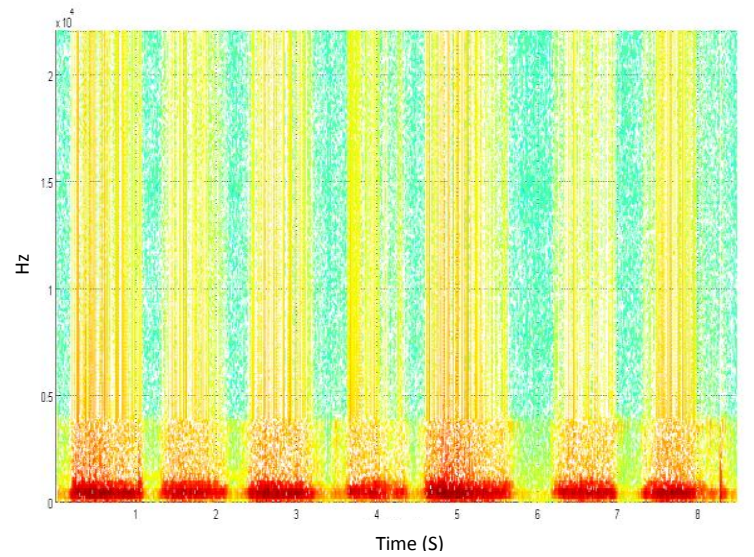

(b)

Fig 4. Having a cold subject: (a) the recorded lung sound, (b) corresponding spectrogram 
previous studies [8-11], the theoretical proof was performed by Fourier transform, as well as the statistical analysis was operated by computer simulation to achieve the box plot of the root-mean-square (RMS) errors from 100 trials for each variable. The sampling rates were 44.1(Group 1), 22.05(Group 2), 11.025(Group 3), 5.5125(Group 4), and 2.76125(Group 5) $\mathrm{KHz}$. The statistical results were presented in Box-andWhiskers display. The input signals were sine waves whose amplitude was equal to 1, and frequencies were 200, 400, 600, $800,1 \mathrm{~K}, 1.2 \mathrm{~K}, 1.4 \mathrm{~K}$, and $1.6 \mathrm{KHz}$. The signal-to-noise ratios (SNR) were $30 \mathrm{~dB}$ for the subjects of normal breath, and 35 $\mathrm{dB}$ for that of wheezing. In summary, we propose the 11.025 $\mathrm{KHz}$ to be fixed sampling rate for noise reduction of wheezing sound recording, and time-variant adjusting sampling rate for the optimal noise cancellation. By the way, the $8 \mathrm{KHz}$ sampling rate of telephone and VoIP has been evaluated to record wheezing sound well. The sampling rate of the study was identified and supported the results of our previous studies [8-11]. In addition the reasons of noises in physiologic signals are usually band-limited, therefore, the optimal sampling frequency can be used to simplify the system design.

The sampling rates of the whole communication processes were listed as follows: The electronic stethoscope was $4 \mathrm{KHz}$ of sampling rate, and the mobile communication was $8 \mathrm{KHz}$ of sampling rate. In our previous studies [8-11], the theoretical proof was performed by Fourier transform, as well as the statistical analysis was operated by computer simulation to achieve the box plot of the root-mean-square (RMS) errors from 100 trials for each variable. The sampling rates were 44.1(Group 1), 22.05(Group 2), 11.025(Group 3), 5.5125(Group 4), and 2.76125(Group 5) KHz. The statistical results were presented in Box-and-Whiskers display. The input signals were sine waves whose amplitude was equal to 1 , and frequencies were $200,400,600,800,1 \mathrm{~K}, 1.2 \mathrm{~K}, 1.4 \mathrm{~K}$, and $1.6 \mathrm{KHz}$. The signal-to-noise ratios (SNR) were $30 \mathrm{~dB}$ for the subjects of normal breath, and $35 \mathrm{~dB}$ for that of wheezing. In summary, we propose the $11.025 \mathrm{KHz}$ to be fixed sampling rate for noise reduction of wheezing sound recording, and time-variant adjusting sampling rate for the optimal noise cancellation. By the way, the $8 \mathrm{KHz}$ sampling rate of telephone and VoIP has been evaluated to record wheezing sound well. The sampling rate of the study was identified and supported the results of our previous studies [8-11].

In addition the reasons of noises in physiologic signals are usually band-limited, therefore, the optimal sampling frequency can be used to simplify the system design.

The signals in Fig. 3, and 4 had been verified by medical doctors of the authors of this study. The confirmed that the sound is good enough for distant application. In this study the electronic stethoscope was seriously selected for a precise acoustic signal to test the experimental system.

\section{CONCLUSION}

This distant electronic stethoscope is a very good tool for the health care. Based on the tests, we believe that the system is reliable. The system can be improved by the management of database for the hospitals' business model. Therefore, our study will invite the medical doctors to authorize the quality of sounds, and will build a server to manage the sound files.

\section{ACKNOWLEDGEMENTS}

The authors thank for the valuable comments from the reviewers and the supports from the project numbers of NSC101-2221-E-562-004, and NSC102-2627-E-002-005, National Science Council, Taiwan, Republic of China.

\section{REFERENCES}

[1] L. A. Geddes, "Birth of the Stethoscope," IEEE Engineering in Medicine and Biology Magazine, vol. 24, pp. 84-86, January-February, 2005.

[2] G. C. N. Meslier and J-L. Racineux, "Wheezes," Eur. Respir. J., vol. 8, pp.1942-1948, 1995.

[3] M. Bahoura, "Pattern recognition methods applied to respiratory sounds classification into normal and wheeze Classes," Computers in Biology and Medicine, vol. 39, pp. 824-843, 2009.

[4] M. J. Murray, Critical care medicine: perioperative management, 2nd ed., Lippincott Williams \& Wilkins, 2002.

[5] C. Kroen, "Back to the Bedside: The Art of Physical Diagnosis," The Hospitalist, pp. 1-39, 22 January/February 2003.

[6] L. Williams and Wilkins, Auscultation Skills : Breath and Heart Sounds, fourth ed., Springer, 2009.

[7] W. Wiebicke, R. Fenton and H. Pasterkamp, "Subjective Assessment vs Computer Analysis of Wheezing in Asthma.," Chest, vol. 91, pp. 376-381, Mar. 1987.

[8] B. S. Lin, H. D. Wu, F. C. Chong, and S. J. Chen, "Wheeze Recognition Based on 2D Bilateral Filtering of Spectrogram," Biomedical Engineering Applications,Basis and Communications, vol. 18, pp. 128-137,Jun. 2006.

[9] B. Y. Lu, J. S. Lai, H. D. Wu, Y. R. Chen, Y. L. Weng, S. H. Tang, Y. L. Chen and L. M. Tseng, "Reduction of the Noise in the Respiration Sound Card: A Preliminary Study," The 15th International Conference on Advanced Communication Technology, Phoenix Park, Republic of Korea, January 27 30, 2013, pp.357-361.

[10] B. Y. Lu, M. N. Hsueh, Y. L. Weng and S. H. Tang, "Reduction of the Noise in the Respiration Sound Recording by the Optimal Sampling Rate of Sound Card: The Verification by Simple Filters," The 15th International Conference on Advanced Communication Technology, Phoenix Park, Republic of Korea, January $27 \sim 30,2013$, pp.148-153.

[11] B. Y. Lu, L. C. Huang, L. M. Hsu, S. H. Tang, H. D. Wu and J. S. Lai, "Statistical Perspective on Noise Cancellations of Wheeze Recordings by Adjusting the Sampling Rates of Sound Card," International Conference on Information Technology and Applications, Australia, Sydney, 2013, pp. 192195. 


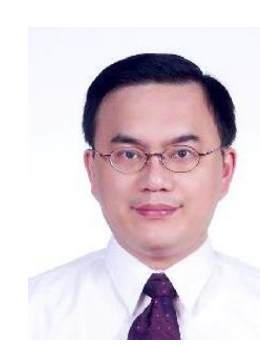

Bing-Yuh Benjamin Lu received his BS degree in electrical engineering from National Central University and his MS and $\mathrm{PhD}$ degrees in electrical engineering from National Taiwan University in 1988, 1993, and 2000, respectively.He was an instructor at the Department of Electronic Engineering, Tungnan University in 1992, and became an associate professor at the same department in 2000. He joined the Department of Information Management, St. Mary's Medicine, Nursing, and Management College, Yi-Lan, Taiwan, Republic of China in 2011, and served as an associate professor and the department head in the duration of 2011 to 2013. He is currently an associate professor in Department of Electronic Engineering, Tungnan University, New Taipei City, Taiwan, Republic of China. He is interested in computer simulation, medical engineering, acoustics, lung sound and educational researches. He is a member of IEEE. His academic interests in focus on acoustics, medical engineering, computer simulation, educational applications of engineering, and pulmonary signal processing.

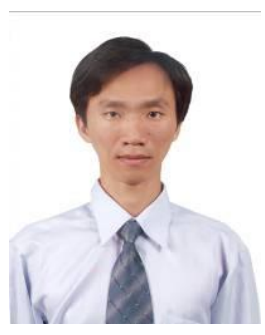

Ling-Yuan Hsu was born in I-Lan, Taiwan, R.O.C., in 1975. He received the M.S. degree in computer science and information engineering from National Dong Hwa University, Hualien, Taiwan, in 2004, and Ph.D. degree in computer science and information engineering at National Taiwan University of Science and Technology, Taipei, Taiwan, in 2013. Currently, he is an assistant professor in the Department of Information Management of ST. Mary's Medicine, Nursing, and Management College. His research interests include artificial intelligence, image processing, evolutionary computation, and computer networks.

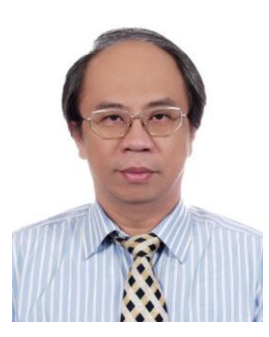

Huey-Dong Wu was graduated with a M.D. degree from Medicine Department of National Taiwan University in 1984. He received resident training in National Taiwan University Hospital (NTUH) and trained as visiting scholar in ULCA later. He service in the Department of Internal Medicine and Department of Integrated Diagnostic \& Therapeutics in National Taiwan University Hospital (NTUH) till now. He is the chief of the division of Respiratory Care, NTUH. His academic interests in focus on respiratory care, pulmonarysiology and pulmonary signal processing especially about lung sound and pulmonary mechanism.

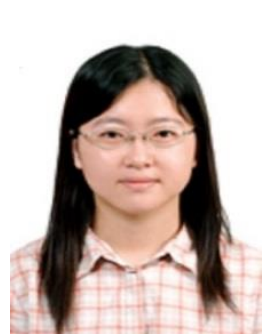

Meng-Lun Hsueh received her BS degree in animal science and MS, and $\mathrm{Ph}$. D. degrees in electrical engineering from National Taiwan University, in 1998, 2002, and 2011 .She has been an instructor of department of electronic engineering, Hwa Hsia Institute of Technology, Taipei, Taiwan. Now, she is an assistant professor, department of electronic engineering, Hwa Hsia Institute of Technology. Her researches include signal processing, and biomedical instrumentation.

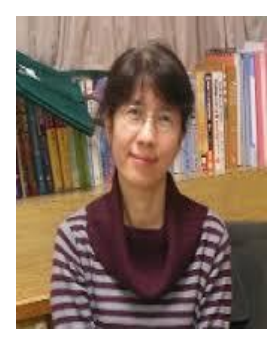

products.

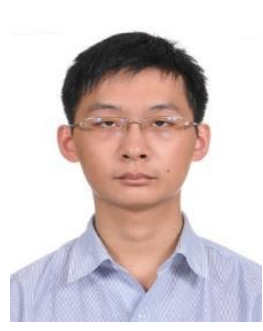

Rui-Han Tang is a college student at Department of Electronic Engineering, Tungnan University, New Taipei City, Taiwan, Republic of China. His major skill is the implement of circuit design.

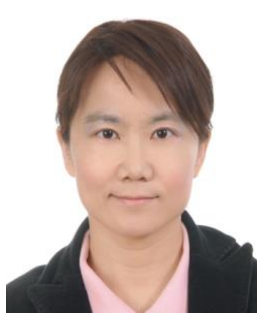

Mei-Ju Su received Ph.D. degree at Graduate Institute of Electronics Engineering, National Taiwan University since 2004 to 2010, Master degree at Department of Electrical Engineering, Syracuse University since 1996 tp 1997. Now, her researches focus on biosignal processing, medical device, telehealthcare system, and sensor design. She had been worked at Industry for 8 years since 1998 2005 on network IC design and SiS Company, medical instrument system design at Biomedical Technology and Device Research Laboratories of Industrial Technology Research Institute. Now she is the associate professor of department of Biomedical engineering, Yuanpei University. 


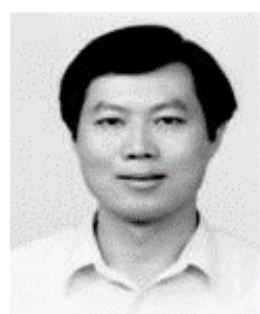

Jhen-Cheng Wang, associate professor in the electronic engineering department of Tungnan University, Taipei, Taiwan. He received master degree of electronic engineering from University of Santa Clara, California, and $\mathrm{PhD}$ degree of mechatronic engineering from Huafan Univerity, Taiwan. His major interests include studies in microprocessors, embedded systems, networks, and networking applications.

Jin-Shin Lai was born in Taipei, Taiwan,

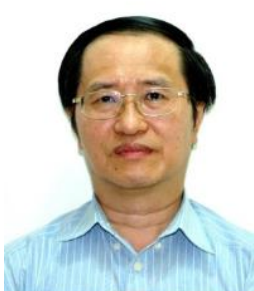
Republic of China (R.O.C.) in 1949. He received the M.D. degree from the Medical School of National Taiwan University, Taipei, Taiwan in 1974. He completed residency training in the Department of Physical Medicine and Rehabilitation, National Taiwan University Hospital, Taiwan in 1978.

He was a Lecturer, Associate Professor, and Professor of Medical School, College of Medicine, National Taiwan University in 1980, 1984 and 1996, respectively. Now, he is the Director of Health Science and Wellness Center, National Taiwan University and Director of Sports Medicine Center, National Taiwan University Hospital. His professional interests include medical informatics, sports medicine, rehabilitation medicine, biomechanics, and rehabilitation engineering. He and his research group had published a series of studies about Physiological Effects of Tai Chi Chuan Training.

Prof. Lai has been the Chairman of School of Rehabilitation Medicine (1988-1992), Chairman of School of Occupational Therapy (1992-1998), Chairman of Department of Physical Medicine \& Rehabilitation (1993-1999), Chief of Division of Medical Informatics (2001-2007), College of Medicine, National Taiwan University, respectively. He also served as the Chairman of Department of Information Technology \& Service, National Taiwan University Hospital (1998-2004), and elected as the President of Rehabilitation Medicine Association of Taiwan (R.O.C.) 1993-1999, the President of Sports Medicine Association of Taiwan (R.O.C.) 1997-2001, the Chairman of Scientific Commission of Asian Federation of Sports Medicine (AFSM) 1990-2000, the Treasurer of Asian Federation of Sports Medicine (AFSM) 2001-2004 and the Chair of HL-7 Taiwan (2005-2009). 